\title{
Saturation in Deuterium retention of CFC graphite targets exposed to beryllium-seeded plasmas on PISCES-B
}

\author{
R. Pugno*, K. Schmid, M. J. Baldwin§, R. Doerner $\S$, \\ J. Hanna§, D. Nishijima §, V. Rohde, J. Roth \\ Max-Planck-Institut für Plasmaphysik, IPP-EURATOM Association, \\ D-85748 Garching, Germany \\ $\S$ University of California, San Diego CA 92093, USA
}

\begin{abstract}
ITER strike-plates are foreseen to be of carbon-fiber-composite (CFC). In this study the CFC bulk deuterium retention in ITER-relevant conditions is investigated. DMS 701 (Dunlop) CFC targets were exposed to plasma in PISCES-B divertor plasma simulator. Samples were exposed to both pure deuterium plasma and berylliumseeded plasma at high fluences (up to $10^{27} \mathrm{~m}^{-2}$ ) and high surface temperature (1070 K). The deuterium contents of the exposed samples have been measured using both thermal desorption spectrometry (TDS) during baking at $1400 \mathrm{~K}$ and ion beam Nuclear Reaction Analysis (NRA). The total deuterium inventory has been obtained from TDS while NRA measured the deuterium depth distribution.

In the analysed fluence range at target temperature of $1070 \mathrm{~K}$, no fluence dependence was observed. The measured released deuterium is $1.4 \cdot 10^{21} \mathrm{~m}^{-2}$. In the case of target exposure with beryllium-seeded plasma no change in the released amount of deuterium was found. The deuterium concentration inside the samples is almost constant until the probed depth of $>>10 \mu \mathrm{m}$, except in the first $1 \mu \mathrm{m}$ surface layer, where it is 5 times higher than in the bulk. No C erosion/redeposition was observed in the Be-seeded plasma cases.

The measured retention, applied to $50 \mathrm{~m}^{2}$ of ITER CFC surface, would imply a tritium saturated value of $0.3 \mathrm{gT}$, much lower than the ITER safety limit of $350 \mathrm{~g}$.
\end{abstract}


PACS: 52.40.Hf

Corresponding author address: Max-Planck-Institut für Plasmaphysik, Boltzmann str. 2, D-85748 Garching, Germany

Corresponding author e-mail: Roberto.Pugno@ipp.mpg.de 


\section{Introduction}

The present ITER design employs beryllium as main chamber wall material and a combination of tungsten and carbon in the divertor. The beryllium will be eroded from the first wall, enter the SOL, and be transported to the divertor where it will interact with the divertor materials.

In the recent years there has been a big effort to the investigate the plasma interaction with ITER first wall materials [1,2], the effects of beryllium deposition/erosion/redeposition on PFC divertor materials and the concomitant formation of mixed-material surfaces. Also, a significant progress has been made in understanding the graphite retention properties and the effects of beryllium plasma contamination on chemical erosion and deuterium retention.

At the PISCES facility, targets of different materials (W,C,Be) are exposed to pure deuterium as well as beryllium-seeded deuterium plasmas, and dependence on plasma parameters and on beryllium concentration is investigated.

Roth [3] has shown that deuterium retention in CFC materials does not reach saturation but the retention increases roughly as (fluence) $)^{0.5}$, whereas in fine grain graphite (FGG) the deuterium retention reaches quasi-saturation when the $\mathrm{D} / \mathrm{C}$ fraction at the surface approach 0.3-0.4. However the above studies were carried out at surface temperature $<730 \mathrm{~K}$ and fluences $<10^{26} \mathrm{~m}^{-2}$. Within the framework of the EU-US collaboration on PISCES-B these data-sets were now expanded by exposing CFC targets to fluences $>10^{26} \mathrm{~m}^{-2}$ at surface temperature of about $1070 \mathrm{~K}$.

\section{Experimental setup}

DSM 701 (Dunlop, $\rho=1.75-1.90 \mathrm{~g} / \mathrm{cm}^{3}$, open porosity $\leq 15 \%$ ) 2D CFC targets, $22 \mathrm{~mm}$ in diameter and $2.8 \mathrm{~mm}$ thick, were exposed to deuterium plasma containing beryllium in the PISCES-B facility [4]. To simulate the ITER main chamber beryllium source, an evaporative neutral atomic beam source was used. The amount of beryllium in the plasma is controlled by changing the temperature of the evaporative cell. A radial spectroscopic view enables measuring the axial distribution of $\mathrm{D}_{\gamma}(434 \mathrm{~nm})$, Be I $(457.3 \mathrm{~nm})$, Be II $(467.3 \mathrm{~nm})$, and CD band (430 nm) lines during the discharge.

The $\mathrm{D}_{\gamma}$ line intensity is used to verify the temporal stability and axial uniformity of the plasma, the Be I line near the target is a measure of the surface beryllium erosion, and the CD molecule band of the carbon chemical erosion. The plasma parameters $\mathrm{n}_{e}$ and $\mathrm{T}_{e}$ are obtained using a double reciprocating Langmuir probe. The beryllium ion density is obtained from the Be II absolute intensity, using the photon efficiency coefficient from the ADAS database [5] for the measured plasma parameters. 


\section{Measurements}

A set of six CFC samples were exposed to similar plasma background conditions, three in pure deuterium plasma (samples CFC3,CFC4,CFC8) and three with beryllium-seeding (CFC5,CFC6,CFC7), see Table 1. Each set was exposed to three different fluences: a lower fluence (LF) of $1 \cdot 10^{26} \mathrm{~m}^{-2}$, a medium fluence (MF) of $3.5-5 \cdot 10^{26} \mathrm{~m}^{-2}$ and a higher fluence (HF) of $1 \cdot 10^{27} \mathrm{~m}^{-2}$. The plasma parameters during the exposures were $\mathrm{T}_{e}=7-13 \mathrm{eV}, \mathrm{n}_{e}=3-4 \cdot 10^{18} \mathrm{~m}^{-3}$, ion flux $=4-6 \cdot 10^{22} \mathrm{~m}^{-2} \mathrm{~s}^{-1}$. The target temperature was $\mathrm{T}_{\text {surface }}=1070 \pm 30 \mathrm{~K}$. The target was biased at $-50 \mathrm{~V}$. The beryllium concentration was in the range of 0.03-0.1\%.

After the target exposure the samples were analysed in-situ using an Auger Electron Spectrometer (AES) to investigate the surface composition. The samples were subsequently removed and taken to a Scanning Electron Microscope (SEM) to analyse the surface morphology. Finally the targets have been split in halves: one-half was analysed at the PISCES-B TDS system and the other half was analysed using ion-beam technique at IPP-Garching.

\section{In-situ surface analysis}

The AES spectra and the correspondent fractional composition of the the target exposed to the beryllium-seeded plasma at the lowest (CFC5) and highest (CFC7) fluence are shown in Fig.1. The almost identical composition of the two samples shows that after a fluence of $10^{26} \mathrm{~m}^{-2}$ the surface is already in equilibrium with the incoming plasma. This is confirmed by the fact that the chemical erosion suppression time, measured by the decay of the CD molecular band emission, for the sample CFC6 is $130 \mathrm{~s}$, much shorter than the $5500 \mathrm{~s}$ exposure of the sample.

This decay time can be compared with the one obtained by the scaling law in [6] which is 195 s, for the same plasma parameters. The scaling law, obtained using measurements of FGG samples, gives, within $50 \%$, a correct estimate for the chemical erosion suppression also for a CFC target. This indicates that only the plasma facing surface of the sample contributes to the chemical erosion and the different morphology and the higher porosity of the CFC do not affect the process of beryllium coating and the formation of the beryllium carbide protective layer.

Figure 2 shows a SEM surface picture of the samples after exposure to pure deuterium plasma and to beryllium-seeded plasma. The surface morphology differs significantly and changes from a lamellae structure for the chemically eroded carbon surface to a peak like structure for beryllium carbide surface. 


\section{Thermal Desorption Analysis}

To measure the deuterium inventory the samples were heated with a linear ramp of $0.31 \mathrm{~K} \mathrm{~s}^{-1}$ to a top temperature of $1400 \mathrm{~K}$, and the desorbed gas gauged in a high pressure quadrupole residual gas analyser.

In Fig. 3 the TDS profiles for the CFC targets exposed to different fluences of pure deuterium plasma (a) and beryllium-seeded plasma (b) are shown. Unfortunately the peak of desorption was not reached at the highest temperature achievable of $1400 \mathrm{~K}$. However the ion beam measurements (see next Sect. and Fig.9) show that the majority of the deuterium has been removed from the sample after TDS, indicating that the TDS measure is a good estimate of the total D inventory.

In Fig.4 the same data are shown, comparing the TDS time trace of pure deuterium plasma and beryllium seeded plasma for the lowest (left) and highest (right) fluence exposure. The onset of desorption for the mixed material surface (beryllium-seeded plasma) is at about $500 \mathrm{~K}$, lower than for pure carbon surface $(700 \mathrm{~K})$ and in agreement with what has been shown in [7].

The total amount of deuterium released (Fig.5) is $(1.4 \pm 0.1) \cdot 10^{21} \mathrm{~m}^{-2}$, independent of the fluence and of beryllium seeding.

The data obtained in CFC can be compared with the previous data from [7] of the saturated retention in FGG for pure deuterium and beryllium-seeded plasmas. In Fig.6 the retentions for different sample temperatures are shown. The beryllium contaminated surface shows a higher retention with respect to pure carbon surface, but the difference decreases with increasing temperature. The deuterium retention in $\mathrm{CFC}$ is about a factor of 2 higher than in FGG at the same temperature.

In Fig.7 the present data are compared with the results of [3]. The previous measurements, carried out at much higher ion impact energy and lower sample temperature, show an increase of retention with fluence. The new measurements show saturation of retention at target temperature of $1070 \mathrm{~K}$, ion impact energies of $50 \mathrm{~V}$ and fluences above $1 \cdot 10^{26} \mathrm{~m}^{-2}$.

\section{Ion beam analysis}

The $\mathrm{D}$ and Be amounts were determined through nuclear reaction analysis (NRA) utilizing the reactions $\mathrm{D}\left({ }^{3} \mathrm{He}, \mathrm{p}\right){ }^{4} \mathrm{He}$ and $\mathrm{Be}\left({ }^{3} \mathrm{He}, \mathrm{p}\right){ }^{11} \mathrm{~B}$ respectively. The samples were initially measured at primary ${ }^{3} \mathrm{He}$ energies of $800 \mathrm{keV}$ and $1200 \mathrm{keV}$ (respectively for $\mathrm{D}$ and Be), which allow to probe for $\mathrm{D} / \mathrm{Be}$ up to approximately $1 \mu \mathrm{m}$ in depth. The protons from the nuclear reaction were collected in a semiconductor surface barrier detector with opening angle of $0.15 \mathrm{sr}$. The detector was covered with a $12 \mu \mathrm{m}$ Mylar foil to stop scattered ${ }^{3} \mathrm{He}$ and ${ }^{4} \mathrm{He}$ particles, allowing only the protons from the nuclear reaction into the detector. Each spectrum was measured with a ${ }^{3} \mathrm{He}$ 
fluence of $10 \mu \mathrm{C}$ which is a good compromise between counting statistics and particle induced release of the $\mathrm{D}$ during the measurement. Measurements performed at different position on a the same target showed that the $\mathrm{D}$ concentration was laterally homogeneous thus using a different spot for each spectra does not influence the calculation of the D areal density. On selected samples the $\mathrm{D}$ depth distribution was measured by scanning the energy of the analyzing $3 \mathrm{He}$ beam. Due to the peaked nature of the cross section for the $\mathrm{D}\left({ }^{3} \mathrm{He}, \mathrm{p}\right){ }^{4} \mathrm{He}$ reaction, different incident ${ }^{3} \mathrm{He}$ probe for $\mathrm{D}$ at different depths. The energy was varied from $690 \mathrm{keV}$ probing only the surface for D to $3200 \mathrm{keV}$ probing for $\mathrm{D}$ at depths of approximately $10 \mu \mathrm{m}$. The details on how to convert the proton intensities detected at each energy into a depth profile are given in[8].

The deuterium inventory is independent of fluence and of Be-seeding, as shown in Fig.8: all the exposed samples have, within the experimental accuracy, the same surface inventory of $(4.5 \pm 0.5) \cdot 10^{19} \mathrm{~m}^{-2}$ (integrated over $1 \mu \mathrm{m}$ ). For the samples where the full energy scan has been done, CFC3 and CFC4 at medium and high fluence and CFC5 at low fluence with Be-seeding, the inventory integrated over the full measured depth $(10 \mu \mathrm{m})$ is $(1.8 \pm 0.1) \cdot 10^{20} \mathrm{~m}^{-2}$.

The sample CFC3-degassed is the target half which has been baked for TDS analysis: it shows that after baking, the $10 \mu \mathrm{m}$ integrated retention is less than $30 \%$ of the original inventory. This indicate that the TDS measurments give a good estimation of the total inventory.

The D depth profiles are shown in Fig.9. A deuterium retention peak is observed at the surface proximity and an almost flat D profile extending to the maximum probed depth. The D inventory obtained by NRA analysis is $10 \%$ with respect to TDS measurments. To account for the whole TDS measured deuterium inventory, the penetration depth of $\mathrm{D}$ has to be $>100 \mu \mathrm{m}$.

\section{Conclusions}

The present study indicates that for high target temperatures, low impact energies and fluences above $1 \cdot 10^{26} \mathrm{~m}^{-2}$, similarly to FGG, CFC reaches saturation. The measured retention indicates a saturation value of $(1.4 \pm 0.1) \cdot 10^{21} \mathrm{~m}^{-2}$. This, applied to ITER, considering a CFC target surface of $50 \mathrm{~m}^{2}$, would imply a tritium saturated value of $1.4 \cdot 10^{21} \mathrm{~m}^{-2} \cdot 50 \mathrm{~m}^{2}=7 \cdot 10^{22}$ atoms, corresponding to about $0.3 \mathrm{gT}$, much lower than the ITER safety limit of $350 \mathrm{~g}$ [9].

\section{Acknowledgements}

The authors wish to acknowledge and thanks for the support and dedication shown by the technical staff of PISCES-B. This work is supported by the US-DOE under the contract DOE DE-FG03-95ER-54301 and the TASK TW5-TPP-CARWBER of the EFDA technology program. 


\section{References}

[1] A.Loarte et al., J.Nucl.Mat. 337-339 (2005) 816

[2] R.P.Doerner et al, J.Nucl.Mat. 337-339 (2005) 877

[3] J.Roth et al., J.Nucl.Mat. 363-365 (2007) 822

[4] R.P.Doerner et al.,J.Nucl.Mat. 290/293 (2001) 166

[5] H.P.Summers et al., Atomic Data and Analysis Structure - User Manual, Rep.JET-IR(94),JET Joint Undertaking, Abindon, 1994.

[6] D.Nishijima et al., J.Nucl.Mat. 363-365 (2007) 1261

[7] M.J.Baldwin et al.,J.Nucl.Mat. 358 (2006) 96

[8] V.Kh.Alimov, M.Mayer, J. Roth, Nucl. Instr. Meth. B234 (2005) 169

[9] G.Federici et al, Nucl. Fusion 41 (2001) 1967 


\begin{tabular}{lllllll}
\hline Sample & $\begin{array}{l}\text { Be plasma } \\
\text { conc. } \\
{[\%]}\end{array}$ & $\begin{array}{l}\text { Fluence } \\
{\left[\mathrm{m}^{-2}\right]}\end{array}$ & $\begin{array}{l}\text { Retention } \\
{\left[\mathrm{m}^{-2}\right]}\end{array}$ & $\begin{array}{l}\text { Retention } \\
(1 \mu \mathrm{m}) \\
{\left[\mathrm{m}^{-2}\right]}\end{array}$ & $\begin{array}{l}\text { Retention } \\
(10 \mu \mathrm{m}) \\
{\left[\mathrm{m}^{-2}\right]}\end{array}$ & $\begin{array}{l}\text { Be conc } \\
(1 \mu \mathrm{m}) \\
{\left[\mathrm{m}^{-2}\right]}\end{array}$ \\
\hline CFC-8 & 0 & $1 \cdot 10^{26}$ & $1.3 \cdot 10^{21}$ & n.a. & n.a. & n.a. \\
CFC-3 & 0 & $5 \cdot 10^{26}$ & $1.5 \cdot 10^{21}$ & $4.7 \cdot 10^{19}$ & $1.8 \cdot 10^{20}$ & 0 \\
CFC-3deg & as above & as above & degassed & $6.4 \cdot 10^{18}$ & $6.0 \cdot 10^{19}$ & 0 \\
CFC-4 & 0 & $1.1 \cdot 10^{27}$ & $1.4 \cdot 10^{21}$ & $4.6 \cdot 10^{19}$ & $1.9 \cdot 10^{20}$ & 0 \\
CFC-5 & $0.045-0.06$ & $1 \cdot 10^{26}$ & $1.5 \cdot 10^{21}$ & $5.9 \cdot 10^{19}$ & $1.7 \cdot 10^{20}$ & $7.1 \cdot 10^{21}$ \\
CFC-6 & $0.03-0.04$ & $3.5 \cdot 10^{26}$ & $1.3 \cdot 10^{21}$ & $4.0 \cdot 10^{19}$ & n.a. & $1.4 \cdot 10^{22}$ \\
CFC-7 & $0.06-0.1$ & $1 \cdot 10^{27}$ & $1.5 \cdot 10^{21}$ & $3.9 \cdot 10^{19}$ & n.a. & $4.4 \cdot 10^{21}$ \\
\hline Table 1 & & & & & &
\end{tabular}

Table 1

Table 1: In the columns are reported: the sample name, the plasma Be concentration by spectroscopy, the fluence to the target, the D retention obtained by TDS, the D retention integrated over the first $\mu \mathrm{m}$ by NRA, the D retention integrated over $10 \mu \mathrm{m}$ by NRA and the Be concentration over the first $\mu \mathrm{m}$ by NRA. 


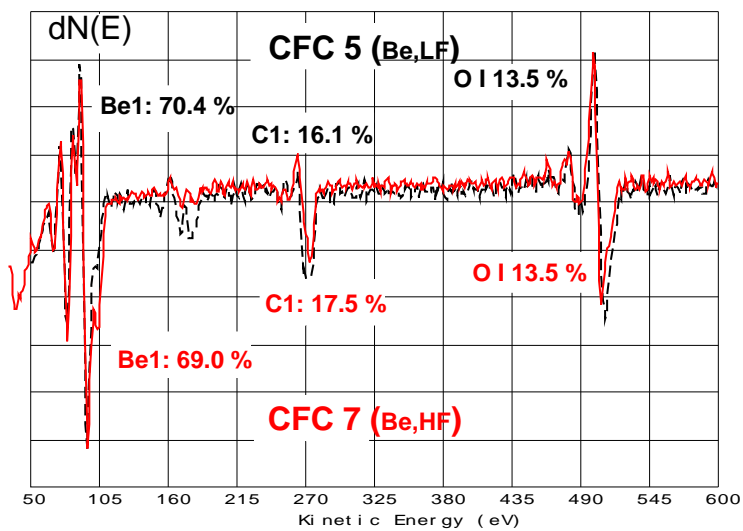

Fig. 1.

Figure 1: Auger Electron Spectroscopy spectrum indicating the surface composition of the CFC target exposed to beryllium-seeded plasma at fluences of $1 \cdot 10^{26} \mathrm{~m}^{-2}$ (CFC5) and $1 \cdot 10^{27} \mathrm{~m}^{-2}$ (CFC7). 

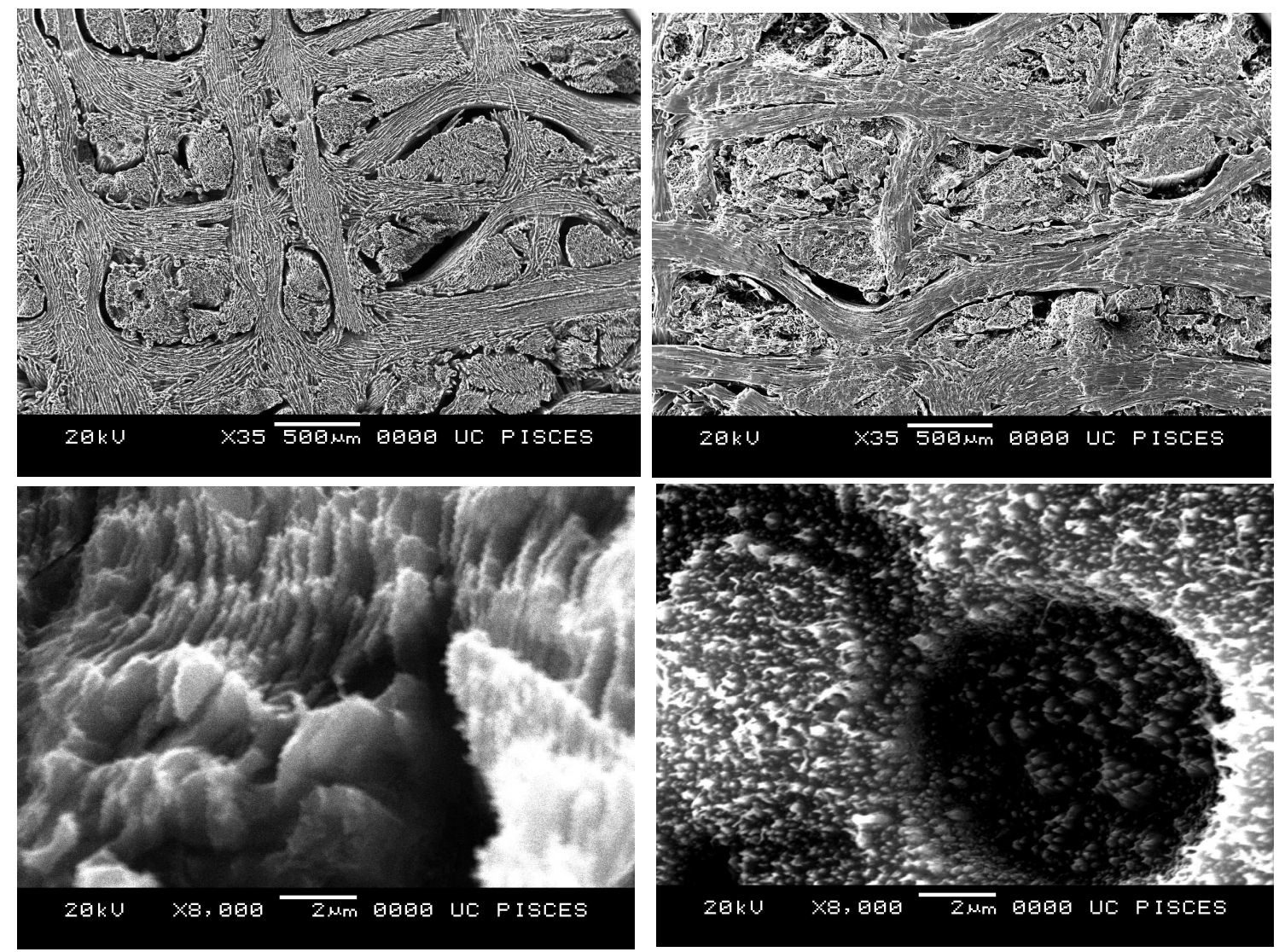

Fig. 2.

Figure 2: Scanning Electron Microscope picture showing the target surface for fluence of $1 \cdot 10^{27} \mathrm{~m}^{-2}$ after exposure to pure deuterium plasma (left) and beryllium-seeded plasma (right). Top and bottom figures show different magnification, as indicated on the picture. The hole in the right-bottom picture is the ending of a CFC fiber perpendicular to the surface plane. 


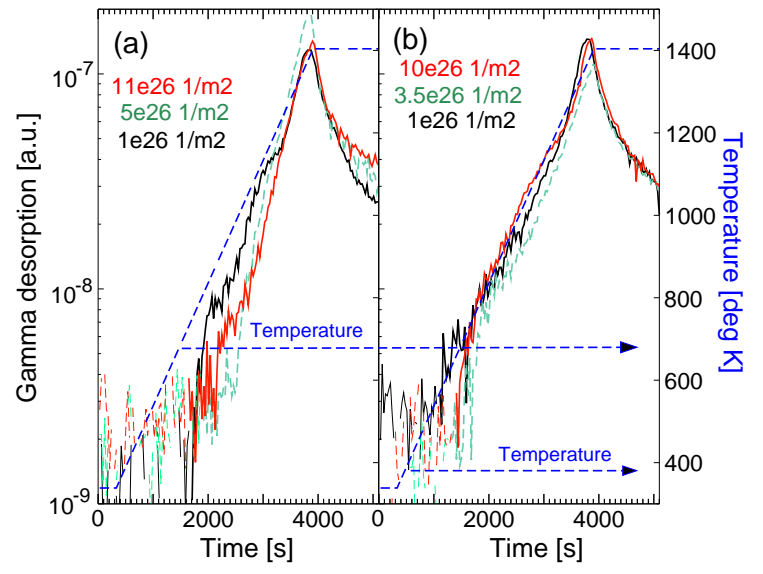

Fig. 3.

Figure 3: Thermal-desorption-spectrometry profile for CFC targets exposed to pure deuterium plasma (a) and beryllium -seeded plasma (b) at different fluences. The temperature ramp is also reported in the figure. 


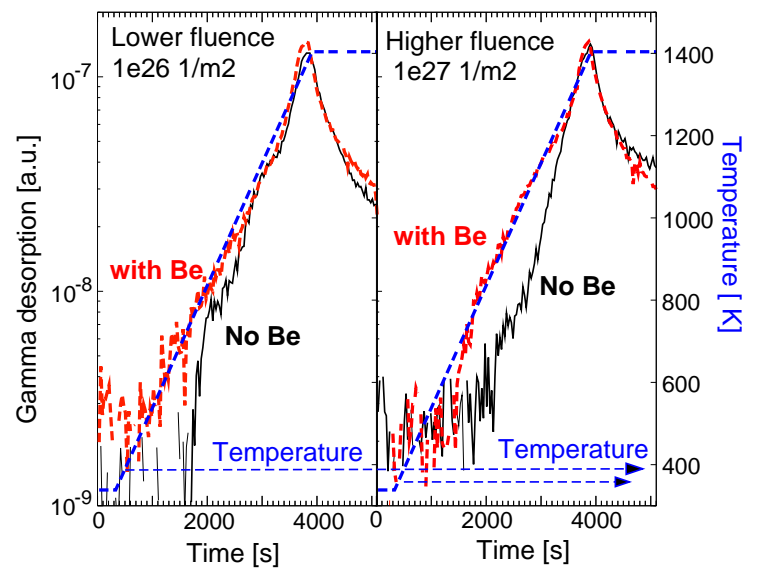

Fig. 4 .

Figure 4: Thermal-desorption-spectrometry profile for CFC targets exposed to pure deuterium plasma at different fluences: (left) $1 \cdot 10^{26} \mathrm{~m}^{-2}$ and (right) $1 \cdot 10^{27} \mathrm{~m}^{-2}$ for pure deuterium and beryllium-seeded plasma. The temperature ramp is also reported in the figure. 


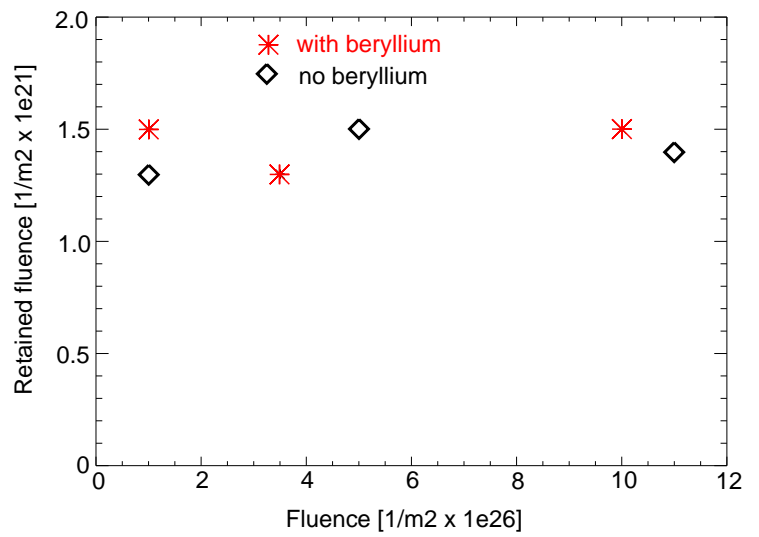

Fig. 5.

Figure 5: Measured deuterium retention for the six exposed CFC samples as function of the ion fluence. 


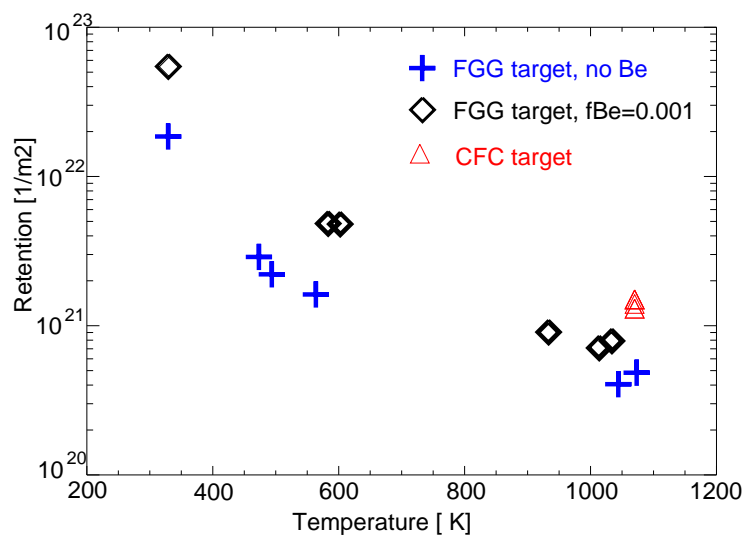

Fig. 6 .

Figure 6: Saturated deuterium retention as function of the target surface temperature. The present measurements in CFC are shown together with earlier measurements in FGG [7]. 


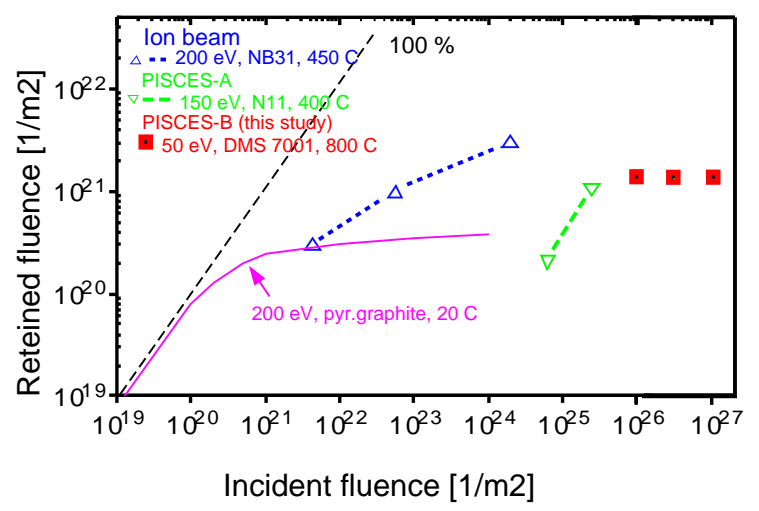

Fig. 7.

Figure 7: Measured deuterium retention as function of the fluence. In this plot, adopted from [3], the results of the present study are also shown. 


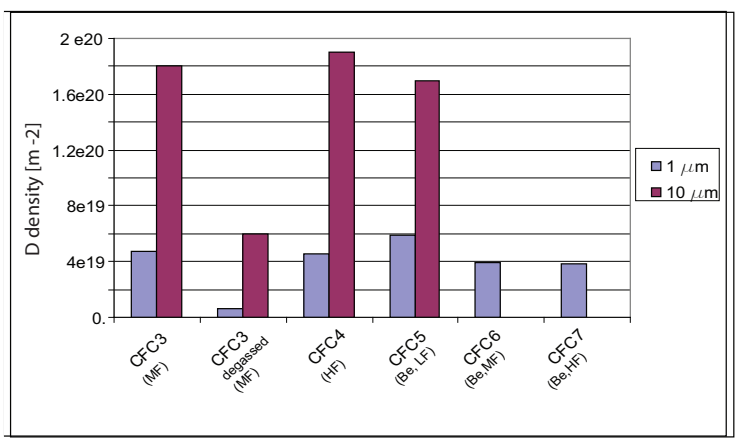

Fig. 8 .

Figure 8: D densities integrated over $1 \mu \mathrm{m}$ surface layer and over $10 \mu \mathrm{m}$ are shown. The sample CFC3-degassed had been baked during the TDS analysis. HF,MF and LF indicate, respectively, the higher, medium and lower fluence cases. 


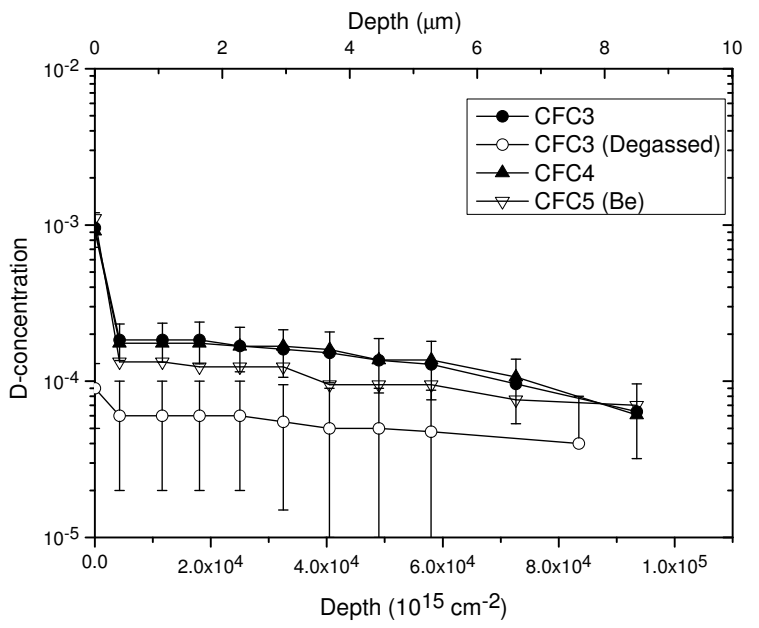

Fig. 9.

Figure 9: Deuterium depth distibution profiles obtained by NRA energy scan. The sample CFC3degassed had been previously baked during the TDS analysis. The error bars for the CFC3 and CFC3-degassed samples are also shown. 